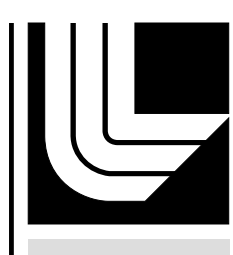

LAWRENCE LIVERMORE N A TIO N A L LABORATORY

\title{
Dynamic Strength of Metals in Shock Deformation
}

\section{UCRL-JRNL-217211}

A. Kubota, D. B. Reisman, W. G. Wolfer

November 21, 2005

Applied Physics Letters 
This document was prepared as an account of work sponsored by an agency of the United States Government. Neither the United States Government nor the University of California nor any of their employees, makes any warranty, express or implied, or assumes any legal liability or responsibility for the accuracy, completeness, or usefulness of any information, apparatus, product, or process disclosed, or represents that its use would not infringe privately owned rights. Reference herein to any specific commercial product, process, or service by trade name, trademark, manufacturer, or otherwise, does not necessarily constitute or imply its endorsement, recommendation, or favoring by the United States Government or the University of California. The views and opinions of authors expressed herein do not necessarily state or reflect those of the United States Government or the University of California, and shall not be used for advertising or product endorsement purposes. 


\title{
Dynamic Strength of Metals in Shock Deformation
}

\author{
Alison Kubota, David B. Reisman, and Wilhelm G. Wolfer \\ Lawrence Livermore National Laboratory, Livermore, California, 94550-9234, USA
}

\begin{abstract}
It is shown that the Hugoniot and the critical shear stress required to deform a metal plastically in shock compression can be obtained directly from molecular dynamics simulations without recourse to surface velocity profiles, or to details of the dislocation evolution. Specific calculations are shown for aluminum shocked along the [100] direction, and containing an initial distribution of microscopic defects. The presence of such defects has a minor effect on the Hugoniot and on the dynamic strength at high pressures. Computed results agree with experimental data.
\end{abstract}

Introduction.- The carriers of plastic deformation in crystalline solids are dislocations. To set them in motion requires shear stresses above a critical value, called the yield or plastic flow strength, or strength for short. It depends on the type of crystal, its orientation with respect to the applied forces, on the temperature, on any previous plastic deformation, and above all on the rate of deformation enforced by the applied forces. In general, the strength increases as the rate of deformation increases. To explore strength at extreme plastic strain rates of $10^{4}$ and beyond, dynamic forces must be applied by impact of solids, by chemical explosives, pulsed magnetic fields, or laser ablation. The rapid application of these extreme forces induce shock waves that propagate from the impacted surface through the material at speeds on the order of the sound velocity, typically several $\mathrm{km} / \mathrm{s}$. Compression of the material and the ensuing plastic flow occur behind the shock front [1]. When the shock reaches the exit surface, its velocity can be measured as a function of time, and from the analysis of this velocity profile, the degree of compression or the density, the pressure, and the dynamic strength can be inferred. However, direct measurements of all three parameters behind the shock front are not possible.

It has therefore been a goal for many years to employ atomistic simulations and study strong shock waves in solids, visualize the dynamic processes during shock compression and deformation, and also "measure", as it were, the thermodynamic field variables such as temperature, density, pressure, flow velocities, and stresses. Holian, one of the prominent pioneers in this field, has reviewed the historical development and the remaining challenges to reach this goal [2]. While the molecular dynamic simulations have provided numerous visualizations of dynamic dislocation behavior during shock compression, the complete set of thermodynamic field variables has so far not been obtained.

Here, we give a brief account of having accomplished this last step, namely a complete mapping of the spatial and temporal distributions of all the thermodynamic variables: temperature, stresses and their rates, strains and their rates, as well as derivative parameters such as pressure, density, von Mises stress, and plastic strain and strain rates. Counting all components of tensors, this is a total of 40 distribution functions. With this information in hand, it is now possible, for the first time we believe, to directly relate pressure to density and temperature on the local scale, and thereby construct the Hugoniot curves of solid and liquid materials, without recourse to the Rankine-Hugoniot relationships. We shall demonstrate this 
below. More significantly, however, is our ability to directly determine the dynamic strength of materials by the inspection of the spatial distribution of the von Mises stress. We consider this as our major breakthrough and the most important result reported in this Letter.

Molecular dynamics of shock compression.- For the present simulations, we have employed empirical interatomic potentials for aluminum, all based on the embedded atom method (EAM) formalism [3]. The supercells employed consist of approximately 8 to 32 million atoms, and are constructed to have an aspect ratio of 4 to 16 based on the longitudinal to transverse dimensions of the lattice. The transverse dimensions were always $32 \mathrm{~nm} \mathrm{x}$ $32 \mathrm{~nm}$. Periodic boundary conditions are applied in the transverse dimensions of the simulation cell, while externally applied forces parallel to the longitudinal direction and corresponding to the desired shock pressure are imposed on $2 \mathrm{~nm}$ thick regions of rigidly associated atoms at the ends of the simulation supercell in the longitudinal direction, designated here as the $\mathrm{x}$-direction. This direction also coincides with the [100] direction of the face-centered-cubic lattice structure. Aluminum crystal supercell are set up devoid of any initial defects, with vacancy-type prismatic loops of $6 \mathrm{~nm}$ diameter and a density of $7.13 \times 10^{17}$ $\mathrm{cm}^{-3}$, with $3 \mathrm{~nm}$ diameter voids of the same density, or with helium bubbles, i. e. the voids filled with 2.2 helium atoms per missing $\mathrm{Al}$ atom. These supercells are then equilibrated at an initial temperature of $300 \mathrm{~K}$ for $10 \mathrm{ps}$, and then normal forces are applied to one of the free surfaces. Piston pressures are thereby generated at 4, 6, 12, 20, and $30 \mathrm{GPa}$. Positions and velocities are computed for all atoms as a function of time and recorded for later processing to produce visual images as well as coarse-grained information. Fig. 1 shows a snapshot at time $40 \mathrm{ps}$ of an aluminum sample with helium bubbles and subject to a piston pressure of 12 $\mathrm{GPa}$; the complete video is available as SOM. The front of the traveling shock is shown as a blue surface. Behind the shock one notices a profuse creation of dislocations. They are visualized by grey atoms which compose the stacking faults between partial dislocations.

Averaging.- The thermodynamic field variables are obtained by taking appropriate averages over fixed volume elements of $1 \mathrm{~nm} \times 2 \mathrm{~nm} \times 2 \mathrm{~nm}$ containing several hundred atoms, the actual number changes with time and location. Detailed spatial and temporal distributions are thereby obtained. In the present Letter we assemble these time-dependent, longitudinal distributions, in so-called x-t plots. Fig. 2 shows an example for four variables, mass density, temperature, pressure $p$, and the von Mises or equivalent stress. The latter two are obtained from the stress components according to the formulae

$$
\begin{gathered}
p(x, t)=-\frac{1}{3} \sum_{y, z}\left(\sigma_{x x}+\sigma_{y y}+\sigma_{z z}\right) \\
\sigma^{e q}(x, t)=\sum_{y, z} \sqrt{\frac{1}{2}\left[\left(\sigma_{x x}-\sigma_{y y}\right)^{2}+\left(\sigma_{y y}-\sigma_{z z}\right)^{2}+\left(\sigma_{z z}-\sigma_{x x}\right)^{2}+6\left(\sigma_{x y}^{2}+\sigma_{y z}^{2}+\sigma_{z x}^{2}\right)\right]}
\end{gathered}
$$

The state of stress is found to vary and does not satisfy the conventional symmetry assumptions that $\sigma_{y y}=\sigma_{z z}$ and $\sigma_{x y}=\sigma_{y z}=\sigma_{z x}=0$, which are based on the uni-axial load application, the parallel orientation of shock propagation and crystal orientation, and on the cubic lattice symmetry. We find invariably that plastic deformation behind the shock front breaks this symmetry, the resulting stress tensor has in general six different components, and the symmetry is broken differently in different volume elements. The $x-t$ plots contain the longitudinal distributions as a function of time; the inverse of the slopes of the left and the 
right borders of the triangular sections are the piston or particle velocity, and the shock front velocity, respectively. The results in Fig. 2 show that the field variables change dramatically, but nevertheless continuously through the shock front, and then approach steady, but not necessarily constant values; small variations sometimes persist over the entire extent of the compressed material. The steady values are taken again as averages, and the ranges of errors as the amplitudes of the variations.

Hugoniot.- Such average values for the pressure are plotted as a function of the average values for the molar volume of aluminum, and displayed in Fig. 3. We also show the Hugoniot curve as obtained by Chisholm et al. [5] from analysis of experimental data and from first principle calculations. Fitting our results of simulations with defect-free aluminum samples gives the dashed curve. It is slightly displaced to the left of the established Hugoniot curve, and indicates that the EAM potential employed needs some refinement. Indeed, we find the pressure derivative of the bulk modulus calculated from this potential is about 3 , while the experimental value is 4.417 [4]. Simulation samples with prismatic dislocation loops give Hugoniot values nearly identical to the defect-free samples. Samples with voids fall on a somewhat lower Hugoniot curve, while samples with over-pressurized helium bubbles are on a somewhat higher Hugoniot curve. The reason for the latter result is that the bulk modulus of helium at the selected helium density in the bubbles is slightly higher than the bulk modulus of aluminum. The softening of the equation of state of a solid by voids and the stiffening by harder inclusions is as expected, and their magnitudes are in full agreement with theoretical estimates. Next, we show the steady temperature reached behind the shock front in Fig. 4 plotted as a function of the pressure $p$, both evaluated over the same region of the sample and the same time. Using the established equation of state for aluminum [5], the adiabatic temperature rise is as shown by the dotted curve. It does not account for the temperature increase generated by plastic deformation, which is of course included in our molecular dynamic simulations. They show again that the microscopic defects affect the temperature rise to a minor degree; samples with voids heat up the compressed material somewhat more, indicating that their collapse under compression generates extra heat.

Dynamic Strength.- We are coming now to the final, and most important finding. The $\mathrm{x}$-t plot for the equivalent stress shown in Fig. 2 reaches a maximum just behind the shock front and then drops off to a steady behavior. Extracting average steady values as well as the amplitude of the quasi-periodic variations, we obtain the results shown in Fig. 5, now plotted as a function of the piston pressure, as it is done for displaying experimental results. These are also shown in Fig. 5 and have been obtained recently by Huang and Asay [6]; they fall approximately within a band indicated by the curves, which serve to guide the eye. The strength values obtained from the molecular dynamics simulations are above this band, more so at lower pressures, but approaching the experimental range at higher pressures. When first noticed, we suspected that the equivalent stress had not actually reached its true steady behavior. As a result, simulations were performed with supercells four times longer. Indeed, as the corresponding data points show, longer supercells are needed to obtain the correct values for steady conditions as the impact pressure is reduced, and these values are then in good agreement with experimental results.

It is important to point out that the strength values extracted from experiments are based on an interpretation of the velocity profile, in particular of the release part of this profile as the shock front is reflected from the free surface opposite to the impacted surface. In contrast, our results are extracted from the actual stress components behind the shock front inside the 
material. The agreement discovered between the simulation and the experimental results is therefore truly remarkable.

The importance of large simulation cells has been found to be crucial for obtaining dynamic strength values that can be compared with experimental results. In our simulations with defect-free samples, the equivalent stress does not approach steady conditions. In fact, it is highly non-uniform as plastic deformation develops only in small isolated parts of the sample, leaving large portions devoid of any dislocations. It may be that the isolated regions of plastic deformation would spread and eventually coalesce in much larger simulation samples. However, based on our observations, the size and the associated time of simulation appear to be beyond the capability of supercomputers expected in the foreseeable future. Fortunately, our discovery reveals that seeding the simulation sample with microscopic defects dramatically reduces both sample size and simulation time to bring it into the realm of present supercomputers. Furthermore, the density of these defects is of the same order of magnitude as the impurity content of readily available pure metals, or of the precipitate density of some aluminum alloys used in commercial and military applications, and also selected by Huang and Asay [6] for some of their experiments. Seeding the simulation samples with defects is therefore not just an artifice but a necessity.

We gratefully acknowledge the support of the Computations Directorate. This work was performed under the auspices of the United States Department of Energy and University of California, Lawrence Livermore National Laboratory, under Contract No. W-7405-Eng-48.

[1] M.B. Boslough and J.R. Asay, High-Pressure Shock Compression of Solids, ed. by J.R. Asay and M. Shaninpoor, Springer Verlag, New York, 1993, chapter 2.

[2] B.L. Holian, High-Pressure Shock Compression of Solids VI, ed. by Y. Horie, L. Davidson, and N.N. Thadhani, Springer Verlag, New York, 2003, chapter 4.

[3] A.F. Voter, A. F., Embedded Atom Method Potentials for Seven FCC metals: Ni, Pd, Pt, $\mathrm{Cu}, \mathrm{Ag}, \mathrm{Au}$ and $\mathrm{Al}$, Los Alamos Technical Report LA-UR 93-3901, 1993.

[4] J.F. Thomas, Phys. Rev. 175, 955 (1968)

[5] E.D. Chisolm, S.D. Crockett, and D.C. Wallace, Phys. Rev. B 68, 104103 (2003).

[6] H. Huang and J.R. Asay, J. Appl. Phys. 98, 033524 (2005). 


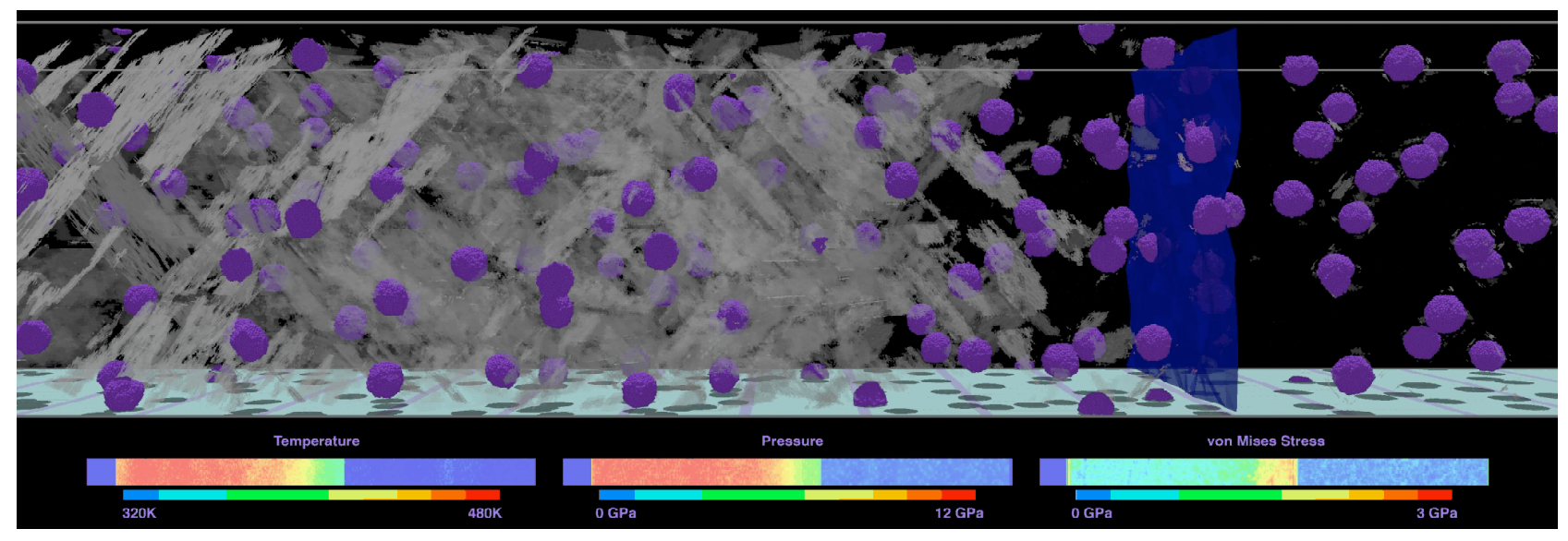

Fig. 1. Snapshot from a MD simulation video of shock propagation through an aluminum crystal containing helium bubbles. The helium atoms are rendered in purple. The front of the elastic precursor is shown as the blue surface moving from left to right at a speed of 6.9 $\mathrm{km} / \mathrm{s}$. Behind the shock, the plastic front is advancing by the generation and expansion of glide dislocations, each consisting of a leading and a trailing partial dislocation. Between each pair, there is a stacking fault in which atoms are rendered in grey. Under the atomic picture are three distributions, for the temperature on the left, for the pressure in the middle, and for the equivalent stress on the right. 

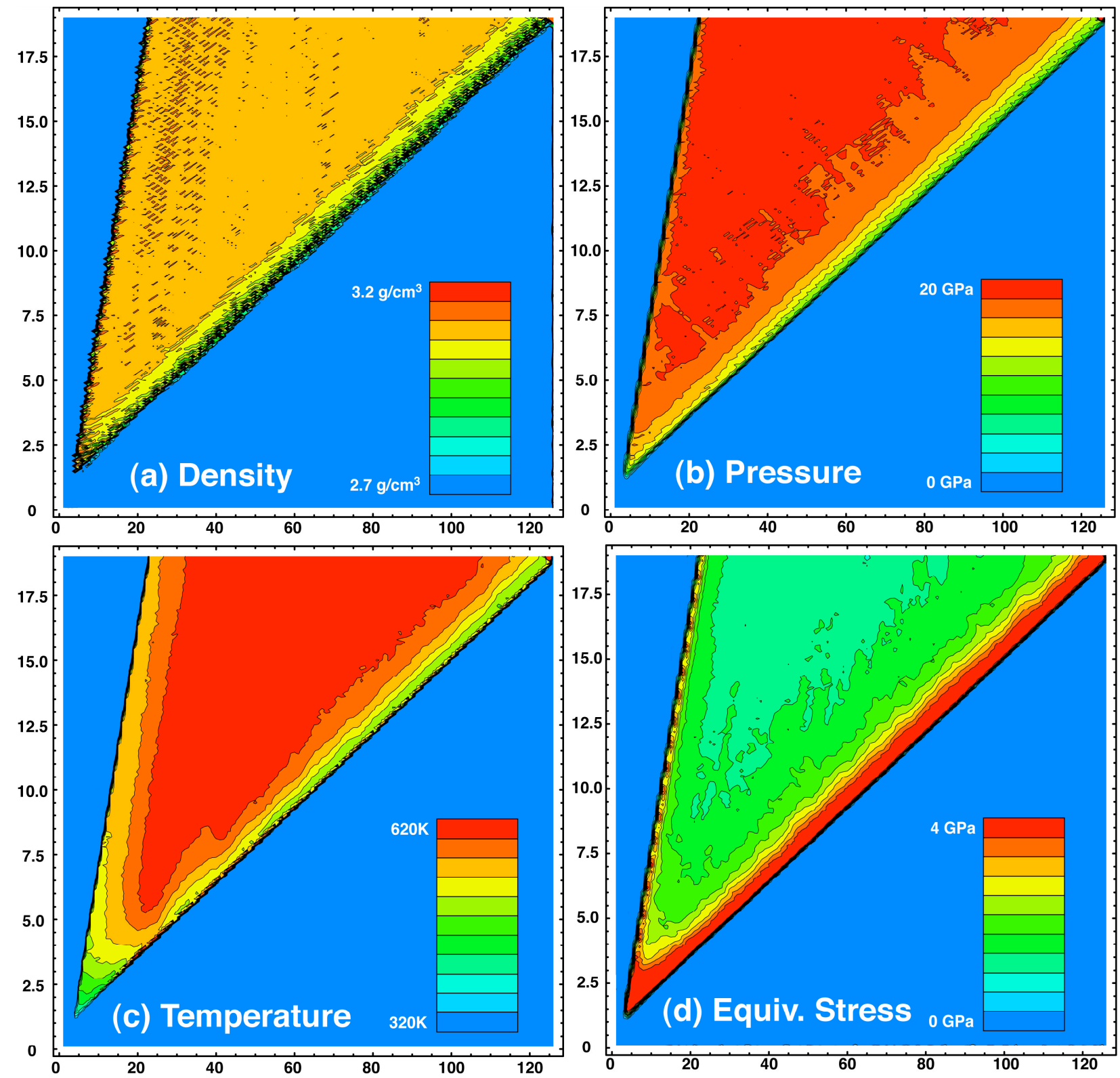

Fig. 2. x-t plots of the spatial and temporal distributions of a) density, b) pressure, c) temperature, and d) equivalent stress, for aluminum with dislocation loops and shocked with a piston pressure of $20 \mathrm{GPa}$. The horizontal axes are in units of $\mathrm{nm}$, the vertical axes are in units of ps. 


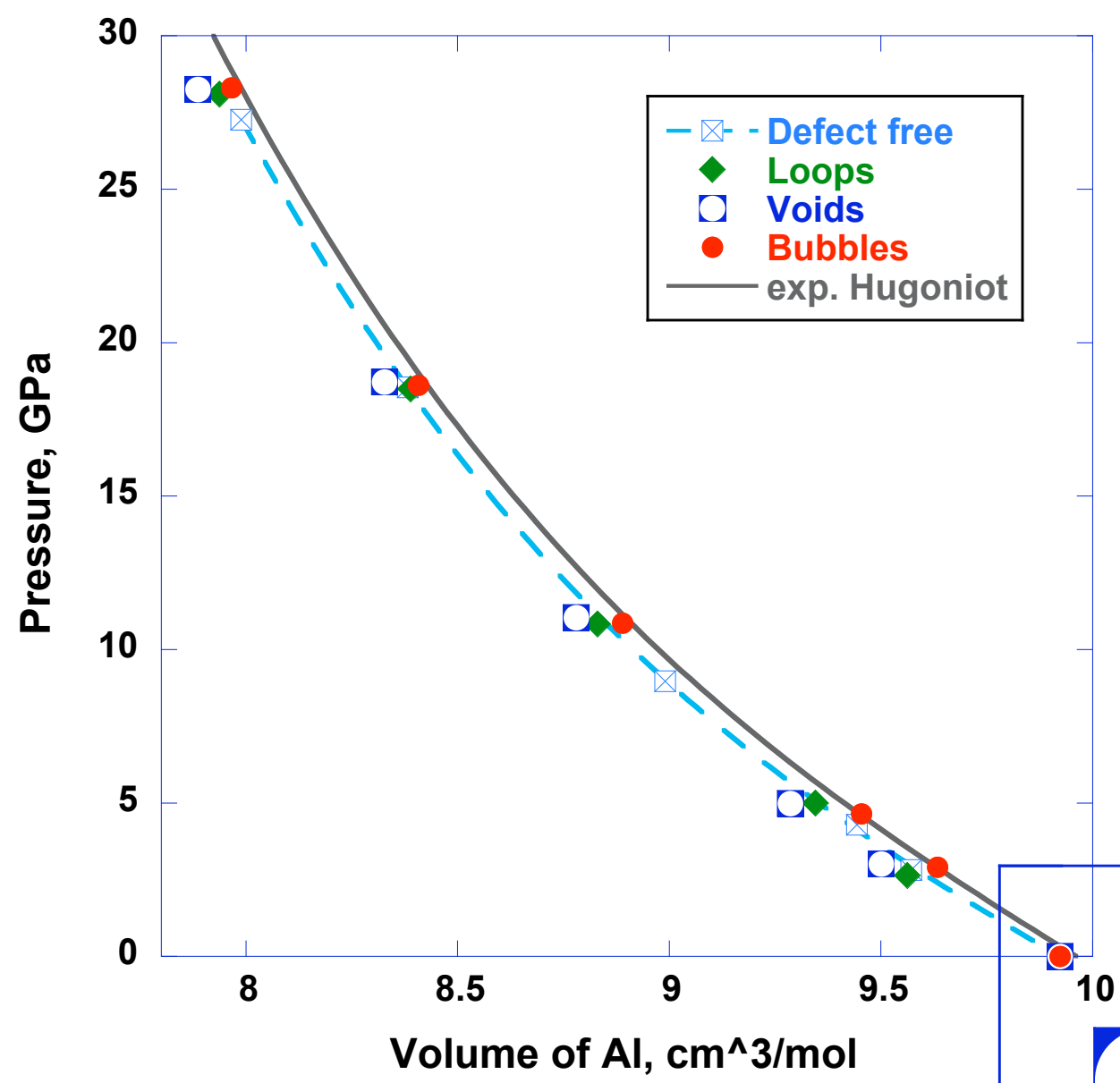

Fig. 3. The Hugoniot of aluminum free of defects and with prismatic dislocation loops, voids, or helium bubbles, as obtained from the MD simulations. The solid curve is the experimentally determined Hugoniot. 


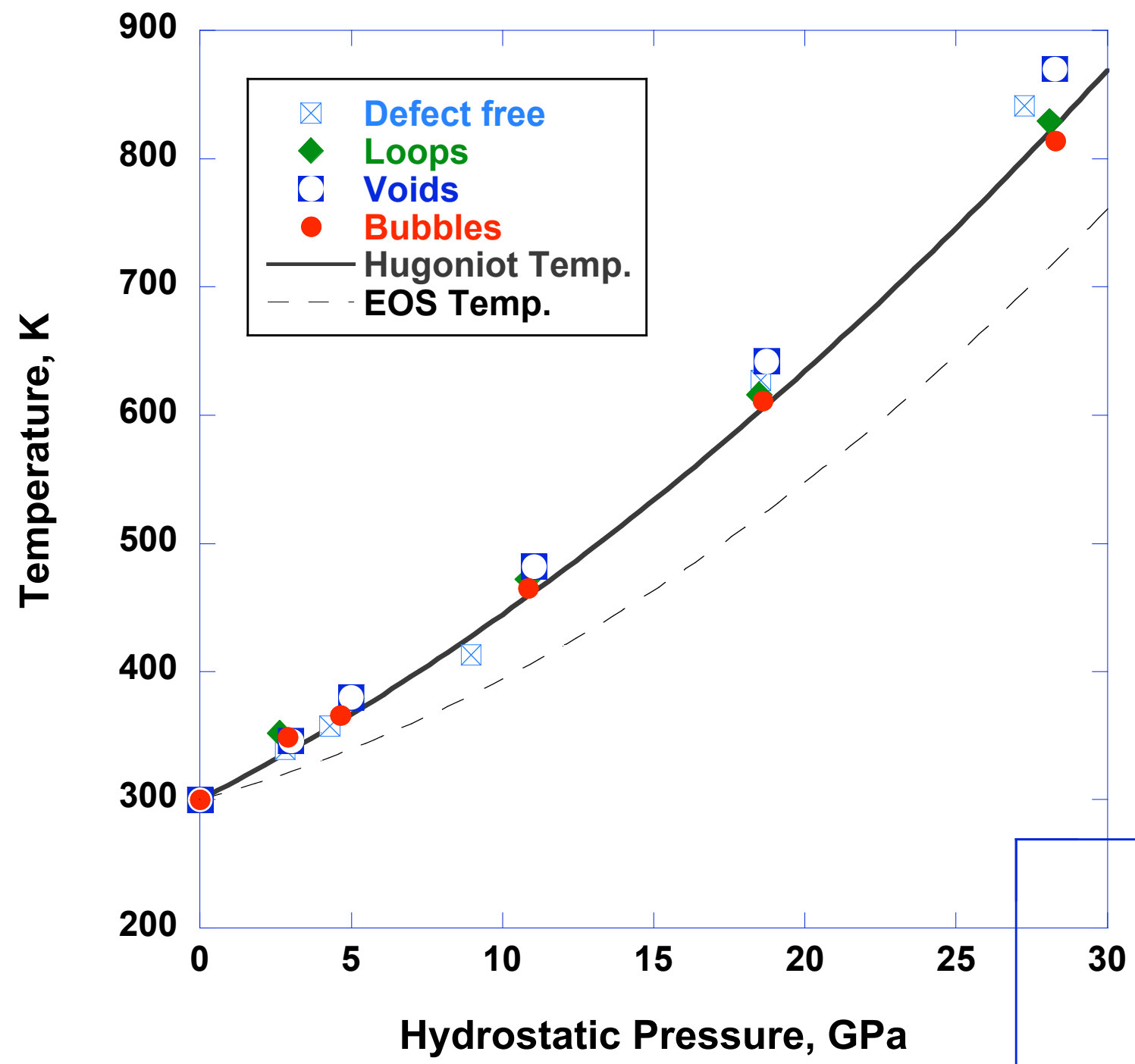

Fig. 4. The steady temperature reached behind the shock front. The solid curve is the Hugoniot temperature obtained from a fit to the results from the defect free simulation samples, and the dashed curve is calculated with the experimentally determined equation of state. 


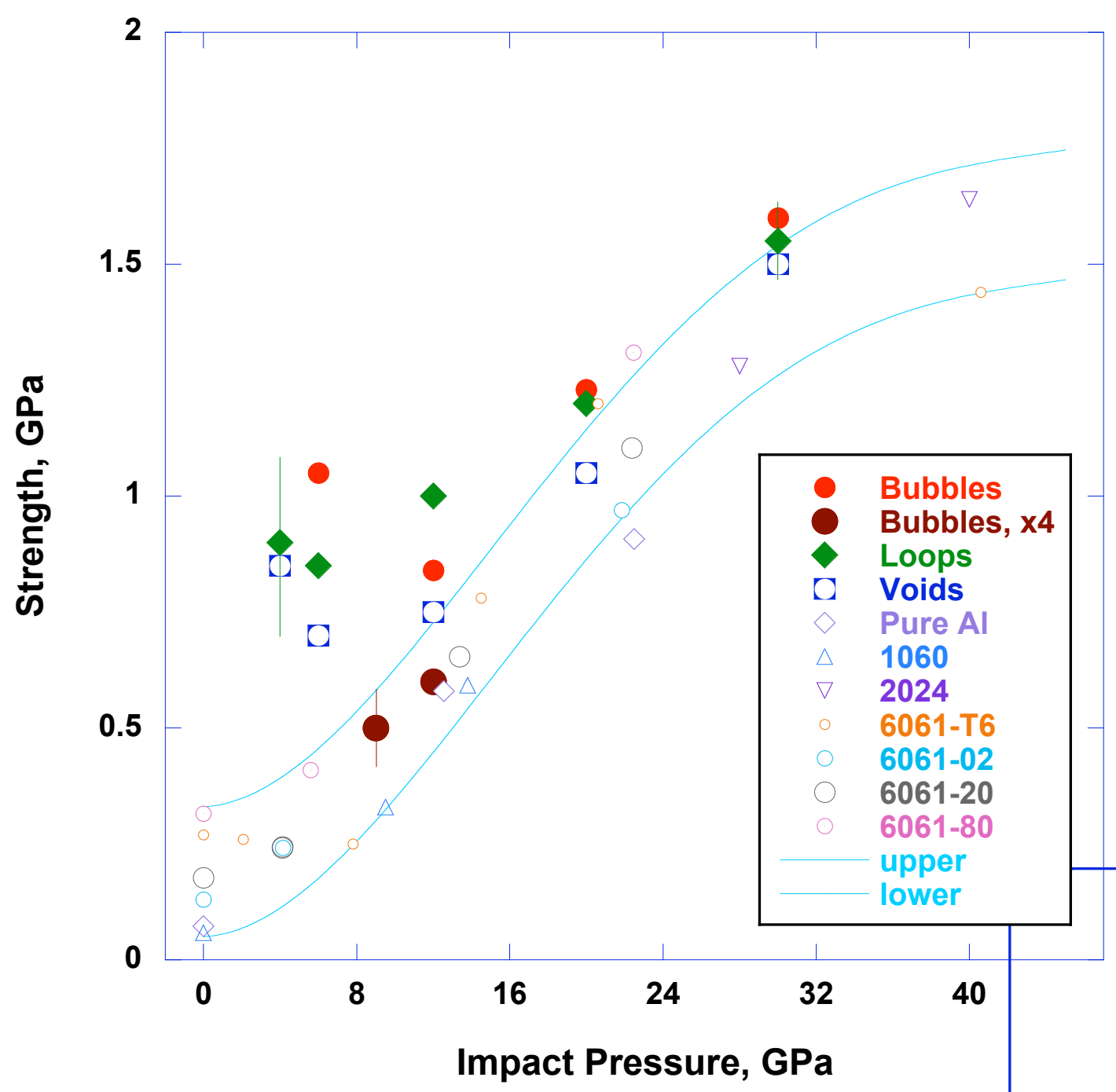

Fig. 5. The dynamic strength values obtained from the simulations (see text), compared with experimental values which fall roughly between the curves. The vertical lines represent error bars. Similar errors apply to the other simulation values, and they are larger at lower impact pressures except for values obtained from simulation cells four times longer. 
